\title{
Evaluation of efficacy of arginine calcium carbonate Vs potassium nitrate paste in the treatment of den- tinal hypersensitivity .
}

\author{
B. Anusha ${ }^{1}$, K. Raja V. Murthy ${ }^{2}$ \\ ${ }^{1}$ Senior Lecturer, Vishnu Dental College, Bhimavaram, Andhra Pradesh, India \\ ${ }^{2}$ Professor and Head of the Department, Department of Periodontics, GITAM Dental College and Hospital, Visakhapatnam, Andhra \\ Pradesh, India \\ Correspondence: boddeda.anusha12@gmail.com
}

\begin{abstract}
Background and Aim: Dentin hypersensitivity $(\mathrm{DH})$ is a common oral health problem affecting one or more teeth of many adult individuals globally. It is characterized by a sharp pain which arises from exposed dentin in response to an external stimulus, and cannot be explained by any other form of dental pathology. Several agents and treatment modalities have been used in the treatment of this condition. The present study was conducted to compare the efficacy of arginine calcium carbonate paste (Test group) versus potassium nitrate (Control group) in the treatment of dentinal hypersensitivity.

Subjects and Methods: A total of 66 cases were randomly divided into two groups based on the toothpaste given: Test group toothpaste containing $8 \%$ arginine and calcium carbonate, Control group - toothpaste containing 5\% potassium nitrate. The patients' DH scores for tactile stimuli was recorded on a visual analog scale and airblast stimuli was recorded on a Schiff Cold Air Sensitivity scale at baseline, immediately post scaling after application of desensitizing paste, 1 month after commencing the use of desensitizing paste, and 1 month following cessation of its use .

Statistical Analysis: The data was analyzed using Independent $t$ test $(\mathrm{P}<0.05)$.

Results: Both the toothpastes were effective in relieving dentinal hypersensitivity. However, toothpaste containing $8 \%$ arginine and calcium carbonate showed a better clinical response at the end of 2 months evaluation.

Conclusions: The desensitizing paste containing $8 \%$ arginine and calcium carbonate showed instant and long lasting reduction in the symptoms of DH.
\end{abstract}

Key Words: Dentinal hypersensitivity, $8 \%$ arginine and calcium carbonate, potassium nitrate, visual analog scale, Schiff Cold Air Sensitivity scale.

\section{Introduction}

Dentinal hypersensitivity $(\mathrm{DH})$ is a short, exaggerated, painful response when opened dentine tubule orifices are exposed to thermal/mechanical/chemical stimuli. This unpleasant feeling is due to stimulation of A-beta fibers and A-delta fibers in the tooth. It is a common clinical symptom with a prevalence rate of $4-57 \%$, seen in adults with age range of 20-49 yrs, with highest incidence at 30-39 yrs and also with female predilection. Hypersensitivity causes include periodontal disease conditions like gingival recession, wasting diseases, faulty brushing and sometimes after flap surgery.[1,2,3]

Although several hypothesis were proposed, Hydrodynamic theory which is based on fluid movement in the dentinal tubules was widely accepted (Brannstrom and Astroem, 1964).Theory states that exposed dentin possess wider tubules with thin/under calcified smear layer, in - creases the centrifugal fluid movement that activates the nerve endings at the end of the dentinal tubules or at the pulp-dentin complex, resulting in sensitivity and unpleasant condition, when compared with unexposed dentin.[4]

Management of DH though well-established has been a challenge to dentist in alleviating the discomfort to the patient. Many methods have been used and still new abrasives are being introduced to market with new ingredients, yet the potentiality of these was questionable in completely eliminating the sensitivity.[5] The management of dentinal hypersensitivity targeted sealing the dental tubules with different molecules/ingredients. Among these commonly used were potassium nitrate and others. Recently, Arginine calcium carbonate was found to be efficient in managing $\mathrm{DH}$.

Till date literature on efficacy of potassium nitrate versus arginine calcium carbonate in alleviating the 
dentinal hypersensitivity was minimal. Hence, this study aims to evaluate the efficacy of these components in managing $\mathrm{DH}$.

\section{Materials and Methods}

This was a single blind, parallel group randomized clinical trial done in the department of Periodontics, GITAM Dental College And Hospital during the period December 2019 till February 2020. Institutional Ethical Committee approval was obtained before the commencement of the study. A total of 66 patients were considered and were randomly divided into control and test group with 33 patients each. Initial examination was done at baseline, and immediately post scaling, following the topical application of abrasive, 1 month after using toothpaste and 1 month after discontinuation of toothpaste.

Patients with age range from 18-70 years with at least two teeth showing hypersensitivity to hot, cold, or sour stimuli were selected. Systemically healthy patients having good periodontal health with probing depth $<3 \mathrm{~mm}$ and no other conditions that might explain their apparent DH were included.

Individuals with chipped teeth, defective restorations, fractured teeth or teeth with deep dental caries, orthodontic appliances, dentures, or bridgework that would interfere with the evaluation of hypersensitivity and who underwent periodontal surgery within the previous 6 months were excluded from the study.[6] Evaluation of hypersensitivity was done using tactile test by visual Ana$\log$ Scale (VAS) and airblast test (Schiff cold air sensitivity scale).[7]

\section{Procedure}

Two different toothpastes, one containing $8 \%$ arginine and calcium carbonate (Colgate Sensitive ProRelief - Colgate-Palmolive Co., New York, NY, USA) (Test group) and another containing $5 \%$ potassium nitrate (Sensodent K - Indoco Remedies Ltd) (Control group) were used in the study.

During the study period, the use of other oral hygiene products or considering any other dental treatment for hypersensitivity was not permitted. Drugs that may alter the perception of pain were not permitted within 24 hours of the assessment. Both the groups after base line evaluation of sensitivity received supragingival scaling with hand instruments. The dentrifice was then applied with fingertip directly on the buccal cervical area of exposed dentin and the sensitivity values were scored after 5 min following application. Each group was provided with that particular dentifrice and were advised to brush their teeth in the Usual manner (modified bass technique) for 3 min, twice daily, with soft bristle toothbrush. They were recalled after 1 month and the sensitivity scores were recorded. Patient was advised to discontinue the prescribed dentrifice and recommended to continue with a fluoridated toothpaste. Thereon patient was asked to report back after one month for the assessment of tooth sensitivity.

\section{Statistical Analysis}

Intergroup comparison of groups with respect to sensitivity scores at different time points was carried out by Independent t-test with significance level was set at $\mathrm{P}$ $<0.05$.

\section{Results}

No adverse effects were observed in any of the cases enrolled in the study. Mean VAS values for tactile stimulus that were assessed for both the pastes (Table 1).

In Test group there was a decrease in sensitivity values from $2.47 \pm 0.28$ at baseline to $1.90 \pm 0.30$ post scaling after application of desensitizing paste. It further reduced to $1.09 \pm 0.42$ after one month of paste use and $1.06 \pm 0.44$ one month following cessation of its use. In Control group there was a decrease in sensitivity values from $2.57 \pm 0.27$ at baseline to $2.21 \pm 0.34$ post scaling. It further reduced to $1.39 \pm 0.34$ after one month of commencing the use of desensitizing paste and was $1.59 \pm$ 0.48 one month following cessation of its use. Mean Schiff Cold Air Sensitivity scale value following air stimulus that were assessed for both the groups (Table 2).

In Test group there was a decrease in sensitivity values from $7.34 \pm 0.41$ at baseline to $6.38 \pm 0.39$ post scaling. It further reduced to $4.97 \pm 0.61$ one month after commencing the use of desensitizing paste and was $4.85 \pm$ 0.59 one month following cessation of its use. In Control group there was a decrease in sensitivity values from 7.66 \pm 0.51 at baseline to $6.93 \pm 0.64$ post scaling. It further reduced to $5.80 \pm 0.61$ after one month of commencing the use of desensitizing paste and was $6.08 \pm 0.67$ one month following cessation of its use.

\section{Discussion}

$\mathrm{DH}$ is relatively a common hitch seen in regular dental practice and is characterized by a sharp, transient unpleasant response to a stimulus like eating, drinking, brushing of teeth, and breathing thus leaving an impact on the quality of life through. As individuals retain their dentitions for longer and as diets change, it is reasonable to 
Table - 1. Comparison of Tactile Sensitivity

\begin{tabular}{|l|l|l|l|}
\hline \multicolumn{1}{|c|}{ Parameters } & Group & \multicolumn{1}{c|}{$\begin{array}{c}\text { Mean } \\
\pm \text { SD }\end{array}$} & $\begin{array}{c}\text { P- } \\
\text { value }\end{array}$ \\
\hline \multirow{2}{*}{ Baseline } & Test & $2.47 \pm 0.28$ & \multirow{2}{*}{0.17} \\
\cline { 2 - 3 } & Control & $2.57 \pm 0.27$ & \\
\hline $\begin{array}{l}\text { After application of } \\
\text { desensitizer }\end{array}$ & Test & $1.90 \pm 0.3$ & \multirow{2}{*}{$<0.01$} \\
\cline { 2 - 3 } $\begin{array}{l}\text { One month of com- } \\
\text { mencing the use of } \\
\text { desensitizer }\end{array}$ & Control & $2.21 \pm 0.34$ & \multirow{2}{*}{$<0.01$} \\
\cline { 1 - 3 } $\begin{array}{l}\text { One month after ces- } \\
\text { sation of desensitizer }\end{array}$ & Cest & $1.09 \pm 0.42$ & $<0.01$ \\
\cline { 2 - 3 } & Control & $1.39 \pm 0.34$ & \\
\hline
\end{tabular}

aints related to dentin hypersensitivity and with that an increase in requests for treatment.[8]

The hydrodynamic mechanism of dentin sensitivity provides a basis for developing desensitizing therapies. The difficulty found in treating $\mathrm{DH}$ is expressed by the enormous number of techniques and therapeutic alternatives to relieve it.[9] According to their mode, treatments can be self administered by the patient at home which is simple, inexpensive and more compliant or can be applied by a dental professional in the dental office. However disadvantages of these treatments include compliance, difficulty in delivery to specific sites, slow onset of action, and requirement of continuous use. Though in-office treatments are more complex and generally target DH localized to one or a few teeth they provide immediate effect might be useful for providing instant relief of hypersensitivity.[10] Hypersensitivity negatively affects the patient's compliance especially during the initial postscaling weeks. ${ }^{7}$ However, patients who have had received periodontal therapy are four times more at risk of developing hypersensitivity than the general population.[8]

Holland et al. in their guidelines for the design and conduct of clinical trials on dentinal hypersensitivity stated that trials should last for at least 8 weeks.[11] The present study also evaluated tooth hypersensitivity over a period of 8 weeks. Furthermore, in the present study, the most and validated stimuli tests for assessing tooth sensitivity, i.e, tactile test and air blast test were used, which are both physiological and controllable. Tarbetet al. and Clark and Troullous stated that these two methods were accurate for the examination of hypersensitivity levels. [12] In the present study, there was a significant decrease in sensitivity scores at all time intervals in both the groups with more alleviated sensivitivity in test group when compared to control group.

Efficacy of Arginine Calcium Carbonate Vs Potassium Nitrate Paste in Dentinal Hypersensitivity 
sensitizing paste to teeth exhibiting sensitivity following dental prophylaxis resulted in instant relief from discomfort and lasted for 28 days after a single application.[17]

In a study by Hamlin et al there is a statistically significant reduction in dentin hypersensitivity compared to a control pumice prophylaxis paste when applied as a single pre-procedural treatment to a professional dental cleaning procedure. They also mentioned that the occlusion led to highly significant reductions in dentin fluid flow by forming a tubule plug which is resistant to normal pulpal pressure.[18]

Potassium nitrate was introduced as a desensitizing agent by Hodosh and it acts by reducing the excitability of the interdental nerves in the pulp by depolarizing them. Potassium ions have to traverse the length of the dentinal tubule in sufficient quantity to cause depolarization.[19] Wilchgers, Ermert and Kim stated that the desensitizing effect of potassium nitrate is due to the increase in concentration of extracellular potassium around the nerve fibres which cause their depolarization, avoids repolarization and blocks the axonic action. This blocks the passage of nerve stimulus, resulting in inactivation of the action potential. Potassium nitrate does not diminish dentine hydraulic conductivity, or promote obstruction of dentinal tubules by the deposition of crystals.[9]

In the present study, there is a decrease in hypersensitivity values following scaling and application of paste in Control group however these sensitivity values were less when compared with test group. There is further decrease in sensitivity after one month of using the paste but the decreased sensitivity values did not sustain after one month of discontinuing the paste. Hodosh stated that topical applications of $1-15 \%$ potassium nitrate (KNO3) or a paste containing $10 \% \mathrm{KNO} 3$ was effective in reducing dentinal hypersensitivity with no pulpal change. 20 A study by Nagata et al revealed that $5 \%$ potassium nitrate performed better than prophylaxis paste at 4,8 and 12 weeks. [21] Purra et al in their study stated that at the end of 5 minutes after treatment, the concentration of potassium ions in the dentinal tubules was not sufficient to make the nerves inexcitable and it takes longer time for the potassium ions to reach the nerves. The effect of desensitization achieved by potassium ions decreased with time and this could be attributed to a decrease in concentration of the ions in dentinal tubules which could the reason for increase in sensitivity after discontinuing the control paste.[19]

In the present study the reduction in sensitivity scores was comparatively more with test group at all time intervals when compared to control group. The results obtained were in accordance with a study by Ayad et al. which showed that the instant sensitivity relief benefit afforded by direct topical self-application of the $8.0 \%$ arginine toothpaste. This was maintained by subsequent regular twice-daily brushing for at least a period of three days performed better than $2 \%$ potassium nitrate and control paste containing 1450 ppm MFP.[22] A study by Elias Boneta et al stated that arginine-containing regimen provided significantly faster dentine hypersensitivity relief compared to the potassium nitrate containing regimen.[2]

\section{Conclusion}

After 2 months of clinical evaluation, both the treatments showed alleviated sensitivity values compared with baseline, independent of their different modes of action. This study demonstrated that the arginine carbonate group (Test group) showed significantly better results compared to potassium nitrate (Control group) by providing instant relief and the obtained relief effect sustained even after discontinuation of paste.

\section{References}

1. Glossary of Periodontal Terms, 4th Edition. 2001.

2. Elias Boneta AR1, Ramirez K, Naboa J, Mateo LR, Stewart B, Panagokos F, De Vizio W. Efficacy in reducing dentine hypersensitivity of a regimen using a toothpaste containing $8 \%$ arginine and calcium carbonate, a mouthwash containing $0.8 \%$ arginine, pyrophosphate and PVM/MA copolymer and a toothbrush compared to potassium and negative control regimens: an eight-week randomized clinical trial. J Dent. 2013 ;41 (1):42-9

3. Mantri V, Maria R, Alladwar N, Ghom S. Dentin Hypersensitivity: Recent Concepts in Management. Journal of Indian Academy of Oral Medicine and Radiology. 2011 Apr 1;23(2):115.

4. Yang ZY, Wang F, Lu K, Li YH, Zhou Z. Argininecontaining desensitizing toothpaste for the treatment of dentin hypersensitivity: a meta-analysis. Clin Cosmet Investig Dent. 2016 Jan 7;8:1-14

5. Birang R, Naghsh N, Yaghini J, Mosavi F. Desensitizing efficacy of Foam Containing Potassium Nitrate 5\% and Toothpaste Containing Strontium Acetate in Dentin Hypersensitivity: An Eight-Week Clinical Study. J Periodontol Implant Dent. 2013;5(1):18-22

6. Pradeep AR, Sharma A. Comparison of clinical efficacy of a dentifrice containing calcium sodium phosphosilicate to a dentifrice containing potassium nitrate and to a placebo on dentinal hypersensitivity: a randomized clinical trial. J Periodontol. 2010 Aug;81(8):1167-73 
7. Pepelassi E, Rahiotis C, Peponi E, Kakaboura A, Vrotsos I. Effectiveness of an in-office argininecalcium carbonate paste on dentine hypersensitivity in periodontitis patients: a double-blind, randomized controlled trial J Clin Periodontol. 2015 Jan;42(1):37-45.

8. Que K, Fu Y, Lin L, Hu D, Zhang YP, Panagakos FS, DeVizio W, Mateo LR. Dentin hypersensitivity reduction of a new toothpaste containing $8.0 \%$ arginine and $1450 \mathrm{ppm}$ fluoride: An 8-week clinical study on Chinese adults. Am J Dent. 2010;23:28A-35A

9. Porto IC, Andrade AK, Montes MA. Diagnosis and treatment of dentinal hypersensitivity. Journal of oral science. 2009;51(3):323-32.

10. Sharma S, Shetty N J, Uppoor A. Evaluation of the clinical efficacy of potassium nitrate desensitizing mouthwash and a toothpaste in the treatment of dentinal hypersensitivity. J Clin Exp Dent. 2012 Feb 1; 4(1):e28-33.

11. Holland GR, Narhi MN, Addy M, Gangarosa L, Orchardson R. Guidelines for the design and conduct of clinical trials on dentine hypersensitivity. J Clin Periodontol 1997;24:808 $\square 13$.

12. Madhavan S, Nayak M, Shenoy A, Shetty R, Prasad K. Dentinal hypersensitivity: A comparative clinical evaluation of CPP-ACP F, sodium fluoride, propolis, and placebo. J Conserv Dent. 2012 Oct;15(4):315-8.

13. Pillai VP, Neelakantan P. Desensitizing toothpastes for treatment of dentin hypersensitivity. Int J Pharm Tech Res. 2013;5(4):1769-73.

14. Lavender SA, Petrou I, Heu R, Stranick MA, Cummins DI, Kilpatrick-Liverman L, Santarpia III RP. Mode of action studies of a new desensitizing dentifrice containing $8.0 \%$ arginine, a high cleaning calcium carbonate system and $1450 \mathrm{ppm}$ fluoride. Am J Dent.2010;23:14A-9A.

15. Schiff T, Delgado E, Zhang YP, DeVizio W, Mateo LR. Clinical evaluation of the efficacy of a desensitizing paste containing $8 \%$ arginine and calcium carbonate in providing instant and lasting in-office relief of dentin hypersensitivity. Am J Dent 2009; 22:8A15A.

16. Yang ZY, Wang F, Lu K, Li YH, Zhou Z. Argininecontaining desensitizing toothpaste for the treatment of dentin hypersensitivity: a meta-analysis. Clin Cosmet
Investig Dent. 2016 Jan 7;8:1-14.

17. Kleinberg I. Sensistat. A new saliva-based composition for simple and effective treatment of dentinal sensitivity pain. Dent Today 2002; 21: 42-7.

18. Hamlin D, Williams KP, Delgado E, Zhang YP, DeVizio W, Mateo LR. Clinical evaluation of the efficacy of a desensitizing paste containing $8 \%$ arginine and calcium carbonate for the in-office relief of dentin hypersensitivity associated with dental prophylaxis. Am J Dent 2009; 22:16A-20A.

19. Purra AR, Mushtaq M, Acharya SR, Saraswati V. A comparative evaluation of propolis and $5.0 \%$ potassium nitrate as a dentine desensitizer: A clinical study. J Indian Soc Periodontol. 2014; 18(4): 466-71.

20. Bartold PM. Dentinal hypersensitivity: A review. Aust Dent J; 2006;51:212 $\square$.

21. Nagata T, Ishida H, Shinohara H, Nishikawa S, Kasahara S, Wakano Y, Daigen S, Troullos ES. Clinical evaluation of a potassium nitrate dentifrice for the treatment of dentinal hypersensitivity. Journal of clinical periodontology. 1994 Mar 1;21(3):217-21.

22. Ayad F, Ayad N, Delgado E, Zhang YP, DeVizio W, Cummins D, Mateo LR. Comparing the efficacy in providing instant relief of dentin hypersensitivity of a new toothpaste containing $8.0 \%$ arginine, calcium carbonate, and $1450 \mathrm{ppm}$ fluoride to a benchmark desensitizing toothpaste containing $2 \%$ potassium ion and $1450 \mathrm{ppm}$ fluoride, and to a control toothpaste with 1450 ppm fluoride: a three-day clinical study in Mississauga, Canada. J Clin Dent. 2009;20(4):11522.

How to cite: Anusha B, Murthy KRV. Evaluation of efficacy of arginine calcium carbonate $V$ s potassium nitrate paste in the treatment of dentinal hypersensitivity. Ind J Clin Res Dent 2020;1(2): 24-29 